\title{
An Automatic Ordering Method for Incomplete Factorization Iterative Solvers**
}

\author{
P. A. Forsyth \\ and \\ W. P. Tang \\ Dept. of Computer Science \\ University of Waterloo \\ Waterloo, Ontario, Canada \\ E. F. D'Azevedo† \\ Oak Ridge National Laboratory \\ Mathematical Sciences Section \\ P.O. Box 2009, Building 9207A \\ Oak Ridge, Tennessee 37831-8083
}

\begin{abstract}
The minimum discarded fill (MDF) ordering strategy for incomplete factorization iterative solvers is developed. MDF ordering is demonstrated for several model son-symmetric problems, as well as a waterflooding simulation which uses an unstructured grid. The model problems show a three to five fold decrease in the number of iterations compared to natural orderings. Greater than twofold improvement was observed ir the waterflooding simulation.
\end{abstract}

\section{DISCLAIMER}

\begin{abstract}
This remrt was prepared as an account of work sponsored by an agency of the United States Goverriment. Neither the United States Gnvernment nor any agency thereof, nor any of their employees, makes any warranty, express or implied, or assumes any legal liability or responsibility for the accuracy, completeness, or usefulness of any information, apparatus, product, or process disclosed, or represents that its use would not infringe privately owned rights. Reference herein to any specific commercial product, process, or service hy trade name, trademark, manufacturer, or otherwise does not necessarily constitute or imply its endorsement, recommendation, or favoring by the United States Government or any agency thereof. The views and opinions of authors expressed herein do not necessarily state or reflect those of the United States Government or any agency thereof.
\end{abstract}

*This work was supported by the Natural Sciences and Engineering Research Council of Canada, by the Information Technology Research Centre, which is funded by the Province of Ontario, and by the Applied Mathematical Sciences subprogram of the Office of Energy Research, U.S. Department of Energy under contract DE-AC05-840R21400 with Martin Marietta Energy Systems, Inc. through an appointment to the U.S. Department of Energy Postgraduate Research Pro jram administen 1 by Oak Ridge Asscciated Universities.

†Department of Computer Science, University of Waterloo, Waterloo, Ontario, Canada N2L 3G1. Present address, Mathematical Sciences Section, Oak Ridge National Laboratory, Oak Ridge, Tennessee 37831-8083, efdazedo@msr.epm.ornl.gov.

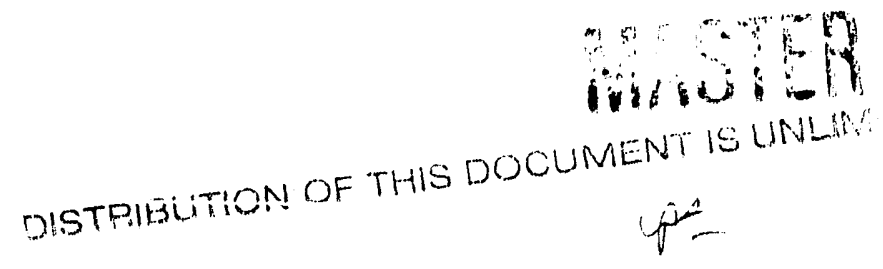


An Automatic Ordering Method for Incomplete Factorization Iterative Solvers

by

E.F. D'Azevedo, P.A. Forsyth and

W-P. Tang

\section{Faculty}

of

\section{Mathematics}

University of Waterloo

Waterloo, Ontario, Canada

N2L $3 G 1$ 
An Automatic Ordering Method for Incomplete Factorization Iterative Solvers

\author{
by \\ E.F. D'Azevedo, P.A. Forsyth \\ and \\ W-P. Tang
}

Research Report CS-90-32

faugust, 1990 


\title{
An Automatic Ordering Method for Incomplete Factorization Iterative Solvers
}

\author{
E.F. D'Azevedo†, P.A. Forsyth*, and \\ W-P. Tang* \\ $\nmid$ Mathematical Sciences Section, \\ Oak Ridge National Laboratory, Oak Ridge, \\ Tennessee. \\ *Department of Computer Science, University of Waterloo, \\ Waterloo, Ontario, N2L 3G1
}

To be presented at the Eleventh SPE Symposium on Reservoir Simulation; Anaheim, 1991. 


\section{ABSTRACT}

The minimum discarded fill (MDF) ordering strategy for incomplete factorization iterative solvers is developed. MDF ordering is demonstrated for several model non-symmetric problems, as well as a waterflooding simulation which uses an unstructured grid. The model problems show a three to five fold decrease in the number of iterations compared to natural orderings. Greater than twofold improvement was observed for the waterflooding simulation. 


\section{Introducticn}

In petroleum reservoirs, the absolute permeabilities are often highly anisotropic and discontinuous. Consequently, the matrices generated in reservoir simulation typically have coefficients which vary by several orders of magnitude. Several authors have noted [1-11] that this has the effect, of making the convergence rate of incomplete factorization methods (ILU) sensitive to the node ordering. Various heuristic methıds have been suggested for alleviating this problem [1-11]. Probably the most successful techinique has been a red-black reduced system method [3-6].

There has been a recent trend towards using highly unstructured grids in reservoir simulation. These types of grids arise in modelling of faults and pinchouts [12], use of local mesh refinement [13], finite volume type grids [14,15], and multiple porosity, multiple permeability systems [16]. It is pnssible to use a generalized red-black [12] ordering method in these situations, but if the average node connectivity is large, the reduced system will not be much smaller than the original system.

The combination of an unstructured grid with a highly hetrogeneous absolute permeabilities makes ILU methods very sensitive to the node numbering. In many cases, there is no obvious "natural" way to order the unknowns.

It is not possible to determine an ordering for an ILU method based solely on the non-zero structure of the matrix. For example, consider the equation

$$
\frac{\partial}{\partial x}\left(K_{x} \frac{\partial P}{\partial x}\right)+\frac{\partial}{\partial y}\left(K_{y} \frac{\partial P}{\partial y}\right)=0 \text {. }
$$

Suppose that this equation is discretized using the usual five point operator. If $K_{x} \gg K_{y}$, an ILU method (for any level $>0$ ) converges faster if $y-x$ ordering is used, than if $x-y$ ordering is specified [11]. This can be explained by noting that the fill terms decay more rapidly for $y-x$ ordering than for $x-y$ numbering [4]. In other words, the error in the ILU decomposition for any given level (greater than level 0 ) is smaller for $y-x$ ordering than for $x-y$ ordering. Of course, both orderings give rise to matrices with identical structures. Clearly, it is necessary to take into account the actual values of matrix entries, not just the non-zero structure.

In this article, we will describe an automatic ordering technique for arbitrary sparse matrices. This method is based on the idea of selecting an ordering which minimizes (locally) the discarded fill in an ILU factcrization. This technique will be demonstrated on some model non-symmetric problems, as well as a waterflooding reservoir simulation problem. 
It is interesting to note that $\mathrm{MDF}(1)$ automatically produces a generalized redblack ordering [11]. If the original matrix is two-cyclic, then this is equivalent to the usual red-black reduced system approach with an $\mathrm{MDF}(0)$ reordering of the reduced system. This technique also correctly orders an anisotropic system (equation (1)) [11].

In a previous article [11], MDF ordering was tested on a wide variety of symmetric matrices, with discontinuous and anisotropic coefficients, similar to an IMPES pressure equation. It was found that the MDF ordering was never worse than "natural" ordering, and in some instances reduced the number of iterations required for convergence by a factor of four to five $[11]$.

Some model non-symmetric examples are given in the following. As well, we will also demonstrate the efficient use of MDF ordering for adaptive implicit reservoir simulation.

\section{Convection Diffusion Equation With Upstream Weighting}

The first test problem is the convection diffusion equation

$$
P_{x x}+P_{y y}-\beta P_{x}-\gamma P_{y}=-q
$$

which is discretized on the unit square with mesh size $h$ in the following way:

$$
\begin{array}{r}
\frac{\left(P_{i+i, j}-P_{i, j}\right)}{h^{2}}+\frac{\left(P_{i-1, j}-P_{i, j}\right)}{h^{2}} \\
+\frac{\left(P_{i, j+1}-P_{i, j}\right)}{h^{2}}+\frac{\left(P_{i, j-1}-P_{i, j}\right)}{h^{2}} \\
-\min \left(\beta_{i, j}, 0\right) \frac{\left(P_{i+1, j}-P_{i, j}\right)}{h^{2}} \\
-\max \left(\beta_{i, j,}, 0\right) \frac{\left(P_{i, j}-P_{i-1, j}\right)}{h^{2}} \\
-\min \left(\gamma_{i, j}, 0\right) \frac{\left(P_{i, j+1}-P_{i, j}\right)}{h^{2}} \\
-\max \left(\gamma_{i, j}, 0\right) \frac{\left(P_{i, j}-P_{i, j-1}\right)}{h^{2}} \\
=-\frac{q_{i, j}}{h^{2}} .
\end{array}
$$


A second problem was also tested using a more complex velocity field. All parameters used were the same as previously, except that

$$
\begin{array}{lc}
\beta=+50 & \\
\gamma=+100 & j=n y / 2, \ldots, n y \\
\beta=-100 & i=1, \ldots, n x / 3 \\
\gamma=+100 & j=1, \ldots, n y / 3 \\
\beta=+100 & \\
\gamma=-100 & \text { for all remaining nodes. }
\end{array}
$$

The results for this problem are shown in Table 2. Again, the MDF ordering shows a dramatic reduction in the number of iterations. The $\mathrm{MDF}(0)$ ordering is shown in Figure 3. While it might be possible to guess the correct ordering for the simple constant velocity case, the complex velocity profile results is a very complicated ordering pattern. Figure 4 shows the ordering for $M D F(1)$. Note the checkerboard red-black type initial ordering.

These examples show that the MDF technique produces an intuitively correct ordering in simple cases, and also gives good results in terms of a decrease in the number of iterations, for more complex situations.

\section{Convection Diffusion Equation with Central Weighting}

For this example, equation (3) was discretized with central weighting on the convective term:

$$
\begin{aligned}
& \frac{\left(P_{i+1, j}-P_{i, j}\right)}{h^{2}}+\frac{\left(P_{i-1, j}-P_{i, j}\right)}{h^{2}} \\
+ & \frac{\left(P_{i, j}+P_{i, j}\right)}{h^{2}}+\frac{\left(P_{i, j-1}-P_{i, j}\right)}{h^{2}} \\
& -\frac{\rho_{i, j}}{2 h}\left(P_{i+1, j}-P_{i-1, j}\right)-\frac{\gamma_{i, j}}{2 h}\left(P_{i, j+1}-P_{i, j-1}\right)
\end{aligned}
$$


original grid was post-processed using a Delauney type edge swap [20-22] to minimize the number of negative transmissibilities. After edge swapping, the fraction of negative transmissibilities was $<0.5 \%$. The resulting grid shown in Figure 5 consists of 2301 nodes. Adaptive implicit timestepping [23-26] was also employed.

After the edge swapping was performed, the resulting grid was then imported back into the grid generation package, and the numbering was "optimized". This ordering was designed to minimize the handwidth for a direct solver. Since this would be fairly typical of the node numbering normally used for a grid of the type shown in Figure 5 , this orlering was designated as the natural ordering.

In general, there are two unknowns per node (pressure $P$ and saturation $S_{w}$ ), and consequently the Jacobian is a block matrix. Rather than apply MDF ordering to the entire matrix, we adopt a simpler strategy.

The prassure can be considered to be an elliptic or parabolic-like variable, while the saturation is hyperbolic-like. As a result, it is expected that the convergence rate of the iterative solver will be dominated by the pressure variables. This behaviour has been confirmed by the performance of the combinative iterative method [4].

Figure 6a shows a typical block line of the Jacobian. After multiplying by the inverse of the diagonal (the usual first step in an adaptive implicit method), we can regard the entries marked " $\epsilon$ " in Figure $6 b$ as insignificant as regards ordering. A pressure equation is formed by taking $\epsilon$ to be small, and hence a completely decoupled pressure equation results (Figure 6c) for each node. This process is repeated for all block lines. The MDF ordering is then applied to this reduced pressure matrix. Once the ordering has been determined, the original block matrix is reordered, using this new node numbering. A block reordering is used, so that unknowns associated with each node remain tightly coupled together.

The waterflooding problem was run to a simulated tine of 15 years, and the water saturation map at that time is shown in Figure 7. Several major well changes occurred during course of this run (i.e. producers converted to injectors) in order to provide a severe test for the iterative solver.

Two complete runs were carried out. The first run used a generalized red-black reduced system ILU. All black to red connections were eliminated exactly, while all black-black connections were eliminated to level 1. The generalized red-black ordering was determined using the original natural ordering (as defined by the grid generation software) as a starting point. 
The convection diffusion equation was used to generate a difficult matrix problem by using central weighting on the convective term combined with a non-trivial velocity field. Iterative techniques that failed to converge using natural ordering did converge using MDF ordering.

The use of MDF ordering improved convergence for both Orthomin and CGS acceleration. Clearly, a good ordering is beneficial regardiess of the acceleration method used.

MDF ordering was also demonstrated on a waterflooding problem which used an unstructured grid. The ordering was determined by using a reduced pressure matrix, which is a simple and effective strategy. MDF ordering reduced the total number of iterations by more than a factor of two. The total reordering cost was less than $1.5 \%$ of the total CPU cost.

The MDF ordering method provides an effective technique to reduce a matrix solution cost for incomplete factorization iterative methods. This technique can be applied to IMPES, fully implicit, or adaptive implicit simulators.

The largest reduction in solution cost will be observed in problems having highly anisotropic and heterogerieous permeabilities, or in simulations carried out using highly unstructured grids. 


\section{Acknowledgements}

This work was supported by the National Sciences and Engineering Research Council of Canada and by the Information Research Center funded by the Province of Ontario, and by the Applied Mathematical Sciences subprogram of the Office of Energy Research, U.S. Department of Energy under contract DE-AC05-84OR21400 with Martin Marietta Energy Systems, Inc., through an appointment to the U.S. Department of Energy Postgraduate Research Program administered by Oak Ridge Associate Universities.

The authors would also like to thank R. McLaren of the Waterloo Center for Groundwater Research for providing a copy of the GRID BUILDER package. 
[13] P. Quandalle and P. Besset, Reduction of grid effects due to local sub-gridding in simulations using a composite grid, SPE 13527, Eighth SPE Symposium on Reservoir Simulation, Dallas, 1085.

114] Z.E. Heinemann and C. Brand, Modelling reservoir geometry with irregular grids, Paper SPE 18412, Tenth SPE Symposium on Reservoir Simulation, Houston, 1989.

[15] P.A. Forsyth, A control volume finite element method fo" local mesh refinement, Paper SPE 18415, Tenth SPE Symposium on Reservoir Simulation, Houston. 1989.

[16] K.H. Coats, Implicit compositional simulation of single porosity and dual porosity reservoirs, Paper SPE 18427, Tenth SPE Symposium on Reservoir Simulation, Houston, 1989.

[17] P. Sonneveld, CGS, A fast Lanczos-type solver for nonsymmetric linear systems, SLAM J. Sci. Stat. Comp. 10 (1989) 36-52.

[18] P.K.W. Vinsome, Orthomin, an iterative method for solving sparse sets of simultaneous linear equations, Paper SPE 5739, Fourth SPE Symposium on Reservoir Simulation, Los Angeles, 1976.

[19] R.G. McLaren, Grid Builder User's Guide, Waterloo Center for Groundwater Research, 1990.

[20] R. Sibson, Locally equiangular triangulations, Computer J. 21 (1978) 243-245.

(21] E. Joe, Delauney triangular meshes in convex regions, SIAM J. Sci. Stat. Comp. 7 (1986) 514-539.

[22] P.A. Forsyth, A control volume finite element method for NAPL groundwater contamination, submitted to SLAM J. Sci. Stat. Comp.

[23] G.W. Thomas and D. Thurnau, Reservoir simulation usong an adaptive implicit method, Soc. Pet. Eng. J., 23 (1983) 759-768.

[24] P.A. Forsyth and P.H. Sammon, Practical considerations for an adaptive implicit method in reservoir simulation, J. Comp. Phys. 62 (1986) 265-281.

[25] T.F. Russell, Stability analysis and switching criteria for adaptive implicit methods based on the CFL condition, Paper SPE 18416, Tenth SPE Symposium on Reservoir Simulation, Houston, 1989.

[26] P.A. Forsyth, Adaptive implicit criteria for two phase flow with gravity and capillary pressure, SLAM J. Sci. Stat. Comp. 10 (1089) 227-252. 
TACLE 1

Results for convection diffusion problem, upstream weighting, constant velocity field.

\begin{tabular}{|l|c|c|c|c|}
\hline \multicolumn{4}{|c|}{ Number of Iterations } \\
\hline \hline & \multicolumn{2}{|c|}{ CGS } & \multicolumn{2}{c|}{ Orthomin } \\
\hline \hline Level of IIU & Natural Ordering & MDF & Natural Ordering & MDF \\
\hline \hline ILU(0) & 17 & 3 & 28 & 5 \\
RS/ILU(0) & 8 & 2 & 7 & 4 \\
\hline
\end{tabular}


TABLE 3

Results for convection diffusion problem, central weighting, complex velocity field.

\begin{tabular}{|c|c|c|c|c|}
\hline \multicolumn{5}{|c|}{ Number of Iterations } \\
\hline & CGS & & Orthomin & \\
\hline Level of ILU & Natiral Ordering & MDF & Natural Ordering & MDF \\
\hline $\mathrm{RS} / \mathrm{ILU}(0)$ & $* * *$ & 36 & **** & 60 \\
\hline
\end{tabular}

*** did not converge in 200 iterations 
FIGURE 1

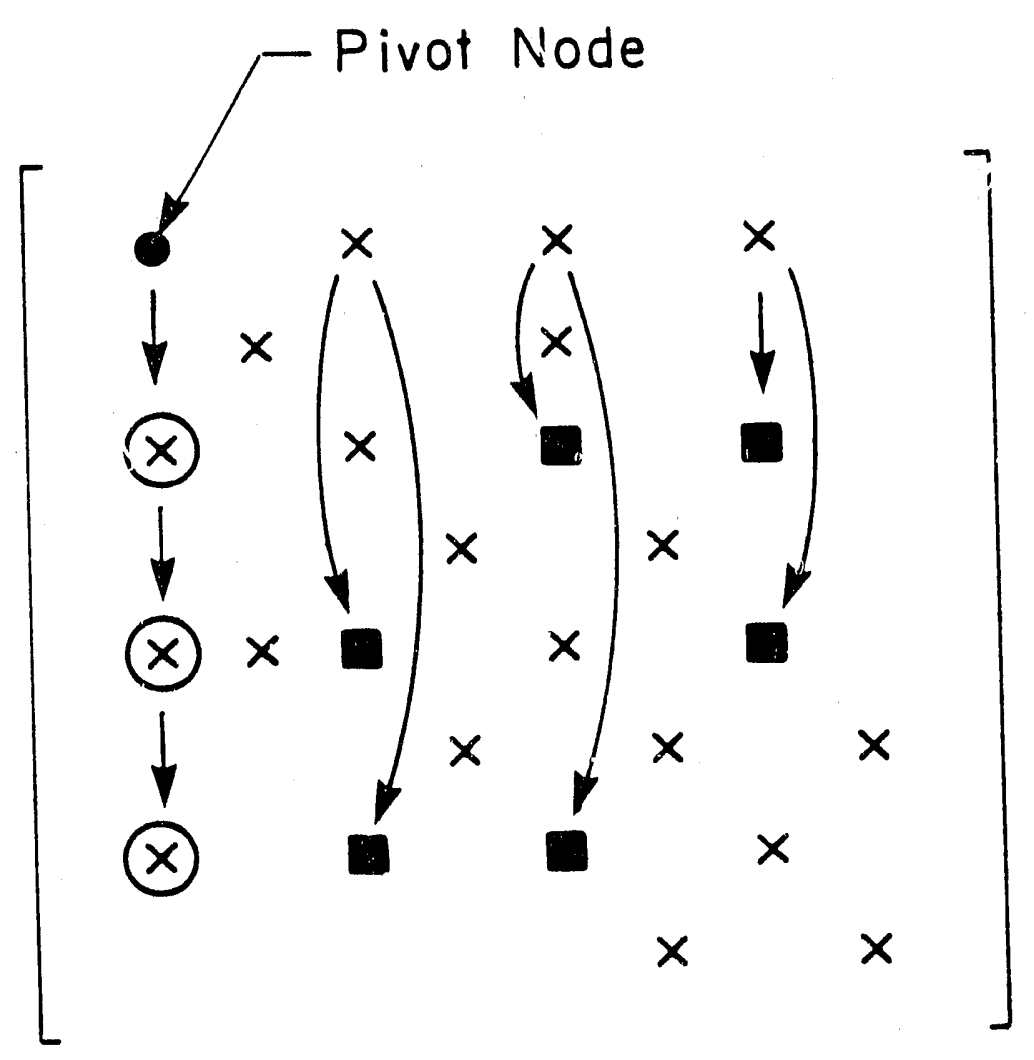

$$
\begin{aligned}
& x \text { - Original matrix entries } \\
& \otimes \text { - Eliminated entries } \\
& - \text { - Level (O) discarded fill }
\end{aligned}
$$

Representation of Level ( 0 ) discarded fill. 


\section{FIGURE 3}

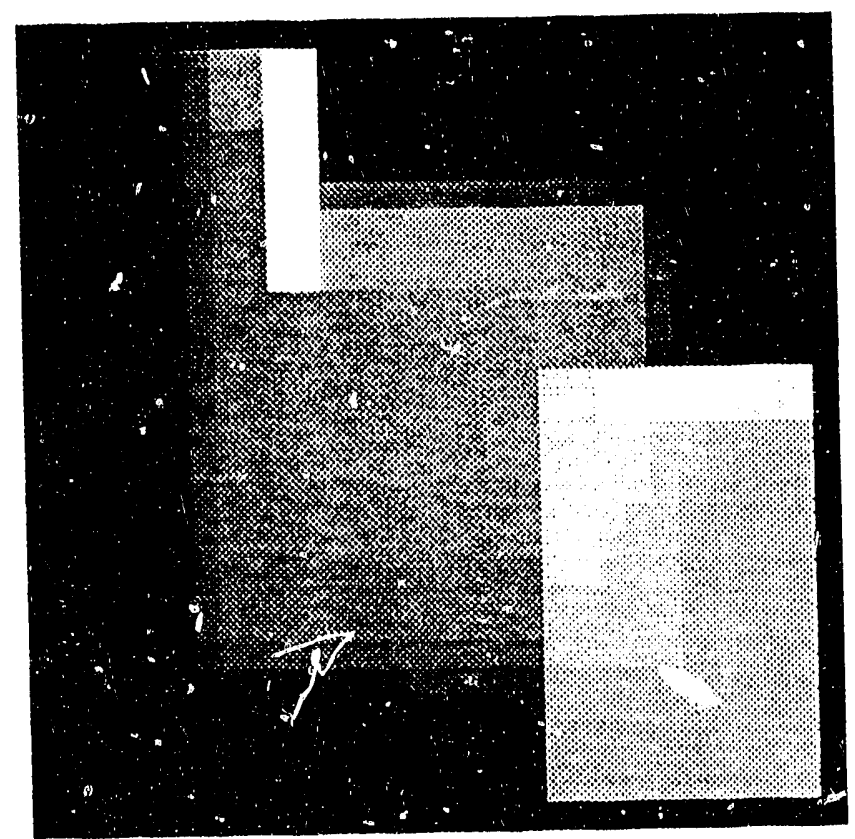

Ordering for $\operatorname{MDF}(0)$, upstream convection diffusion problem, complex velocity field. Darkest-first; lightest-last. 
FIGURE 5

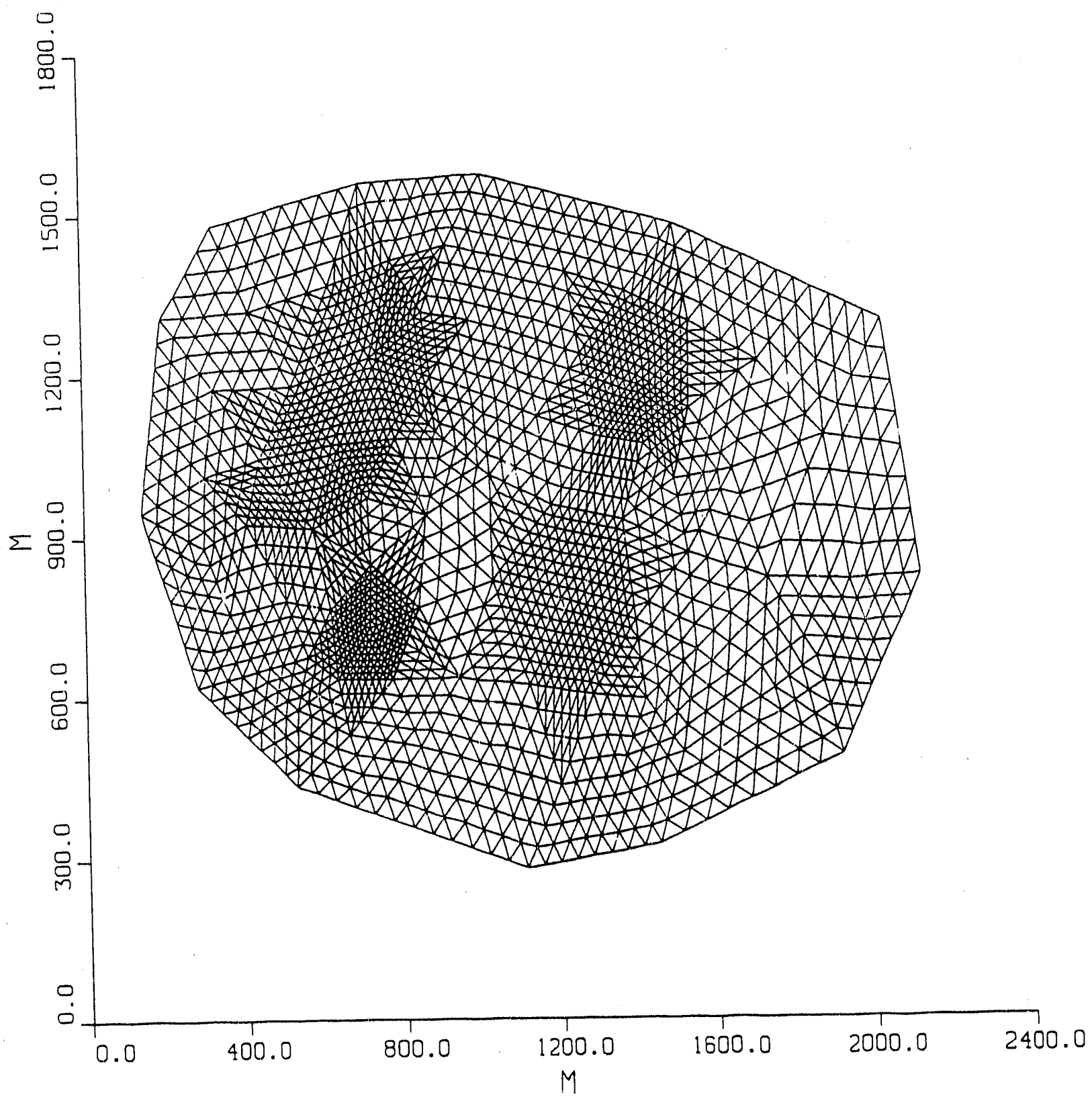

Grid for waterflooding problem. 


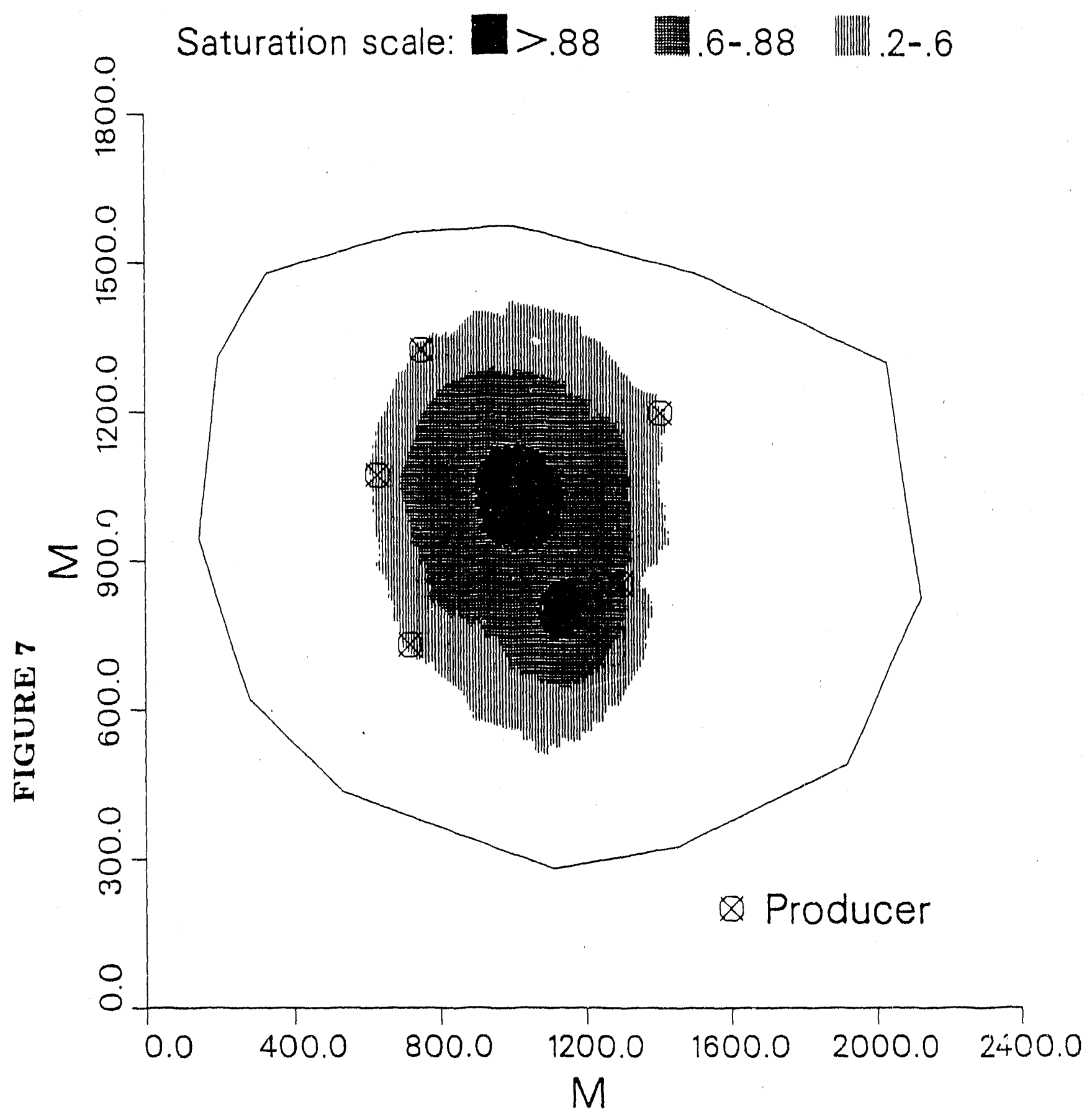

Water saturation values for the waterflooding simulation, time $=15$ years. 

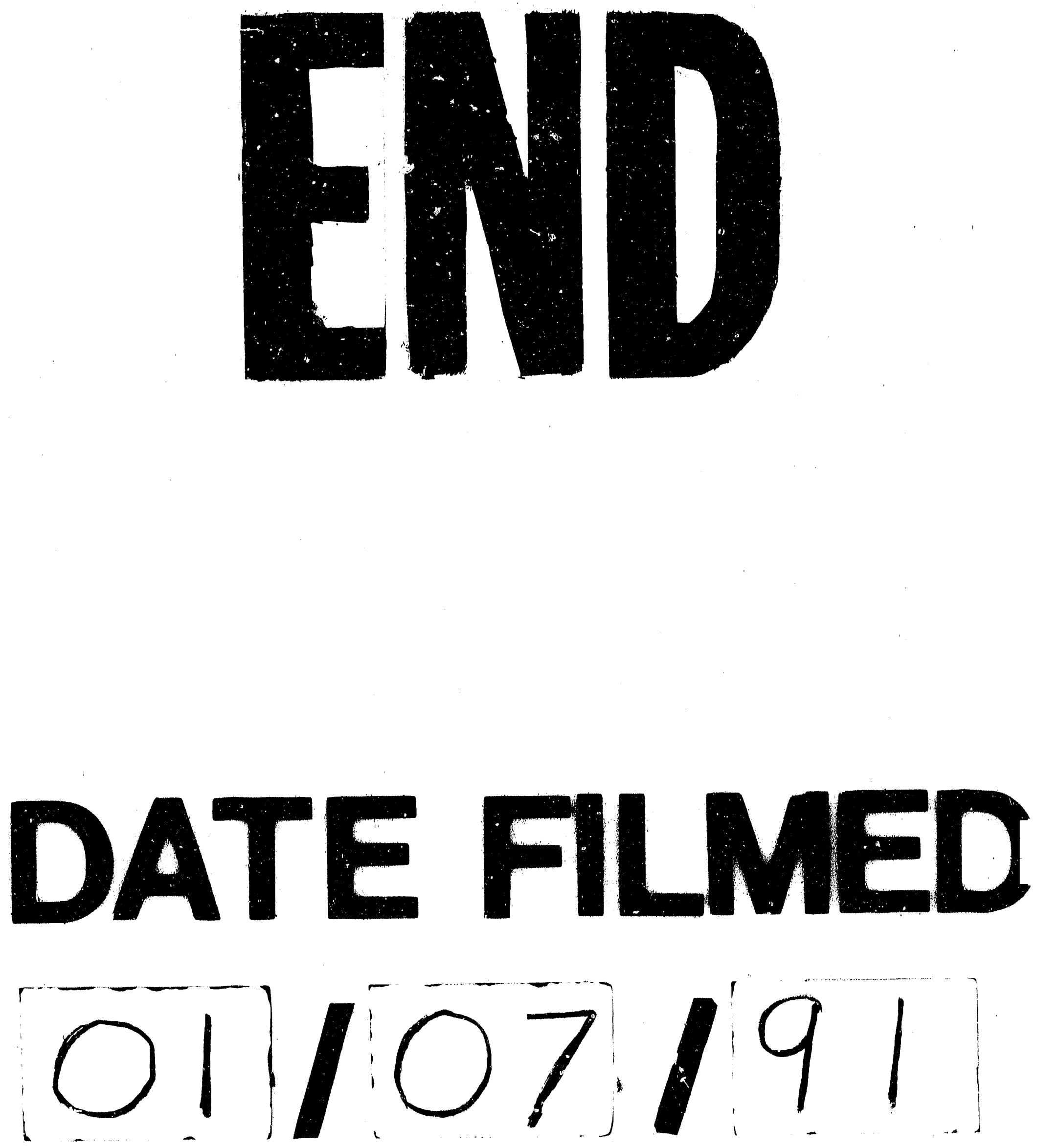
